\title{
Befreiung aus der gesetzlichen Rentenversicherung für jeden?
}

\author{
Mediziner, die sich von der Beitragspflicht zur gesetzlichen \\ Rentenversicherung befreien lassen wollen, müssen berufs- \\ inhaltlich klar ärztlich tätig sein, so ein Sozialgericht.
}

Ärzte haben nicht automatisch das Recht, sich von der gesetzlichen Rentenversicherung befreien zu lassen. Entscheidend ist die jeweils ausgeübte Tätigkeit - bei einem Jobwechsel kann das Befreiungsrecht auch wieder verloren gehen. Ärzte, die im Zweifel sind, ob bei ihnen Befreiungsgrundlagen vorliegen, sollten sich bei der zuständigen Ärztekammer oder der Arbeitsgemeinschaft der Berufsständischen Versorgungseinrichtungen (ABV) erkundigen. In Falle einer Juristin hatte das Sozialgericht Düsseldorf (SG) in einem nicht rechtskräftigen Urteil entschieden, dass die Frau keinen Anspruch auf Befreiung von der gesetzlichen Rentenversicherung hat, obwohl sie Mitglied im Versorgungswerk der Rechtsanwälte ist. Die Juristin arbeitet in der Anspruchsprüfung einer Versicherung und ist zusätzlich als Rechtsanwältin tätig. Das SG gab der gesetzlichen Rentenversicherung Recht, die der Frau die Befreiung verweigert hatte, weil sie bei der Versicherung keine typisch anwaltliche Tätigkeit ausübt. Auch bei Ärzten ist es nicht selbstverständlich, dass sie die gesetzliche Rentenversicherung verlassen können. „Es geht um die konkrete Tätigkeit und nicht um die Approbation", erläutert Dr. Gerhard Rosler, Geschäftsführer der Nordrheinischen Ärzteversorgung (NÄV). „Man muss der Rentenversicherung gegenüber sehr gut beschreiben, was man tut", sagt Rosler. Bei der Prüfung, ob es sich um eine typisch ärztliche Tätigkeit handelt, achtet die Rentenversicherung unter anderem auf die Stellenausschreibung oder die Funktionsbeschreibung. „Richtet ein Unternehmen eine Stellenausschreibung an Chemiker, Ärzte oder Tierärzte, wird es oft schwierig", weiß Rosler. Auch wenn der Arbeitsvertrag nicht ausdrücklich auf die ärztliche Ausbildung und die ärztliche Tätigkeit Bezug nimmt, und die Funktionsbeschreibungen keinen ausdrücklich medizinischen Hintergrund haben, steht die Befreiung von der gesetzlichen Rentenversicherung in Frage. Wenn ein Mediziner innerhalb eines Unternehmens die Stelle wechselt und zum Beispiel reine Management-Aufgaben übernimmt, muss er eine neue Prüfung der Befreiungsgrundlagen beantragen. Sind dann ärztliche Kenntnisse nicht mehr ausschlaggebend, entfallen die Grundlagen für die Befreiung. Die Versorgungswerke ABV klären die Ärzte und auch Unternehmen sorgfältig über die Hintergründe der Befreiungsmöglichkeiten auf, sagt er. Die NÄV stellt - wie andere Versorgungswerke auch - ein Merkblatt zum Befreiungsrecht zur Verfügung. (Ilse Schlingensiepen)

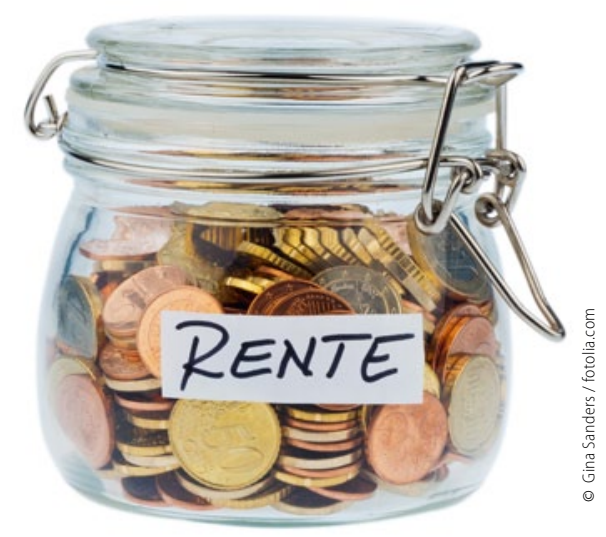

Wer bekommt Rente aus welchem Topf?
Desinfektion: Liste gibt Überblick über geeignete Mittel

Die Bandbreite an Herstellern und Produkten zur Desinfektion in der Praxis ist groß. Auskunft dazu, welche Mittel zum Gebrauch in der Praxis geeignet sind, gibt die Desinfektionsmittel-Liste des Verbundes für Angewandte Hygiene (VAH). In der kostenpflichtigen Liste sind laut VAH alle von der Desinfektionsmittel-Kommision zertifizierten Präparate enthalten. Die Liste soll als Basis für die Auswahl von Desinfektionsmitteln für die routinemäßige und prophylaktische Desinfektion in Krankenhaus und Praxis sowie in öffentlichen Einrichtungen und anderen Bereichen, in denen Infektionen übertragen werden können, dienen. Die Liste enthalte nur Produkte, für die ein Konformitätsbewertungsverfahren durch unabhängige Experten durchgeführt worden ist.

Verbund für Angewandte Hygiene: www.vah-online.de
Schriftform festzulegen. Diese Anforderungen basieren laut KVWL auf dem Infektionsschutzgesetz, der Biostoffverordnung, berufsgenossenschaftlichen Vorschriften (TRBA/BGR 250) und den Richtlinien des Robert Koch-Institutes.

\section{Kontinuierliche Aktualisierung nötig} Der Hygieneplan sei als Sammlung verbindlicher Anweisungen der Praxisleitung an das Praxisteam regelmäßig zu aktualisieren und auf die gegenwärtigen $\mathrm{Ge}$ gebenheiten der Praxis und den jeweiligen Stand der medizinischen Wissenschaft sowie Technik anzupassen, führt die KVWL aus. „Unterweisungen in die Inhalte des Hygieneplans sind bei Bedarf oder mindestens in jährlichen Abständen zu wiederholen und zu dokumentieren. Darüber hinaus liegt es in der Verantwortung jedes Beteiligten in der Praxis, die Inhalte des Hygieneplans zu kennen und umzusetzen“, so die KVWL. Mit der Un- terschrift eines jeden Beschäftigten der Praxis auf dem Hygieneplan verpflichte sich jeder einzelne zur Kenntnisnahme und Umsetzung im Praxisalltag, gibt die KVWL als praktischen Tipp für Praxischefs. Inhaltlich geht es im Hygieneplan dann nicht nur um das klassische Händewaschen und die Händedesinfektion. Der Plan muss auch regeln, ob und wie Medizinprodukte in der Praxis wiederaufbereitet werden und wie mit Patienten umgegangen wird, die Träger von Krankheitserregern wie zum Beispiel MRSA sind. Bei der Flächenreinigung und -desinfektion in der Arztpraxis zum Beispiel geht es auch um Arbeitsmittel wie PC-Tastaturen sowie um das Hygienemanagement in den sanitären Bereichen der Praxis. Die Überwachung und Einhaltung der Lagerfristen von Sterilgut ist ebenfalls im Hygieneplan zu regeln.

(Matthias Wallenfels) 